\title{
Optimizing Panchromatic Image Change Detection Based on Change Index Multiband Image Analysis
}

\author{
E. Martínez, A. Arquero and I. Molina
}

\begin{abstract}
This work proposes an optimization of a semisupervised Change Detection methodology based on a combination of Change Indices (CI) derived from an image multitemporal data set. For this purpose, SPOT 5 Panchromatic images with $2.5 \mathrm{~m}$ spatial resolution have been used, from which three Change Indices have been calculated. Two of them are usually known indices; however the third one has been derived considering the Kullbak-Leibler divergence. Then, these three indices have been combined forming a multiband image that has been used in as input for a Support Vector Machine (SVM) classifier where four different discriminant functions have been tested in order to differentiate between change and no_change categories. The performance of the suggested procedure has been assessed applying different quality measures, reaching in each case highly satisfactory values. These results have demonstrated that the simultaneous combination of basic change indices with others more sophisticated like the Kullback-Leibler distance, and the application of non-parametric discriminant functions like those employees in the SVM method, allows solving efficiently a change detection problem.
\end{abstract}

\section{INTRODUCCION}

$\mathrm{S}_{\mathrm{s}}^{\mathrm{E}}$ E denomina Detección de Cambios (DC) a un proceso mediante el cual se identifican las diferencias en el estado de un objeto o fenómeno al observarlo en diferentes momentos. es decir. se trata de cuantificar efectos temporales utilizando información multitemporal [1]. La determinación de áreas de cambio en imágenes de la misma escena tomada en diferentes momentos, presenta un notable interés, debido a que ofrece un gran número de aplicaciones en diversas disciplinas [2], incluyendo entre otras: la video-vigilancia [3]. el diagnóstico y tratamiento médico. el apoyo a la conducción de vehículos y la detección remota.

Concretamente, la Detección de Cambios en imágenes registradas mediante sensores remotos, está considerada como una rama de la tecnología que constituye la Teledetección. Lo que contribuye a este hecho. es la resolución temporal de los sensores de satélites, cuya inherente repetitividad en su órbita. permite que se puedan registrar con cierta regularidad, imágenes de un determinado área objetivo. Además la continua mejora de la tecnologia espacial ha propiciado el desarrollo de sensores de alta resolución, que proveen de

\begin{tabular}{|c|c|c|c|c|}
\hline $\begin{array}{c}\text { E. Martínez. } \\
\text { emartinezốfifiupni.es }\end{array}$ & Universidad & Politécnica & de & Madrid, \\
\hline $\begin{array}{c}\text { A. Arquero. } \\
\text { aarquero@afiupm.es }\end{array}$ & Universidad & Politécnica & de & Madrid. \\
\hline $\begin{array}{l}\text { I. Molina, } \\
\text { inigo.molina }(\bar{a} \text { upm.es }\end{array}$ & $\begin{array}{l}\text { Universidad } \\
\text { s }\end{array}$ & Politécnica & de & Madrid. \\
\hline
\end{tabular}

grandes volúmenes de datos los cuales a su vez contienen una elevada calidad en su información. En este sentido, en el ámbito de la Teledetección, se han desarrollado muchas técnicas de DC, que se han recopilado en excelentes compendios [1], [2], [4]. [5]. Básicamente, se agrupan según dos enfoques: supervisado y no supervisado [6]. En el primer grupo se incluyen métodos que requieren información de referencia del terreno, necesaria para obtener mapas temáticos de transiciones entre clases de coberturas terrestres que cambian; se basan en el uso de clasificadores supervisados [7]. Esta información de referencia se puede obtener mediante campañas de campo, por fotointerpretación o desde el conocimiento previo de la escena. En estos métodos, como inconvenientes se pueden indicar: el coste humano y temporal que supone la obtención de la información de referencia y el posible error cometido en el proceso de clasificación. Por el contrario, los métodos no supervisados no necesitan datos de referencia para la generación del mapa binario de áreas de cambio/no_cambio. Sin embargo, no se puede identificar en ellos la transición ocurrida entre clases de coberturas de terreno cambiantes. También en ellos, es importante el preprocesamiento de las imágenes [8]. [9]. [10], [11]. porque pueden existir diferencias en el registro de la imagen multitemporal (radiometría y/o geometría de imagen), que producen falsos positivos en los resultados de Detección de Cambios [12].

Cada una de las diferentes propuestas de algoritmos de Detección de Cambios tiene sus ventajas, no habiendo un único enfoque óptimo y aplicable a todos los casos. En la práctica, a menudo, se realizan estudios comparativos entre diferentes enfoques con el objetivo de encontrar los mejores resultados para una aplicación especifica. En cualquier caso, los métodos no supervisados se muestran más atractivos desde el punto de vista operativo, especialmente para el análisis de grandes conjuntos de datos.

Con el fin de aprovechar al máximo las cualidades de las diferentes propuestas de generación de los mapas de cambios, algunos autores [13], [14], sugieren su uso simultáneo y una fusión posterior de las diferentes fuentes de información sobre la presencia o ausencia de cambios en las escenas.

En este trabajo, se propone una metodología semisupervisada de DC mediante la aplicación de métodos no supervisados basados en la diferencia, cociente y cálculo de la distancia de Kullback-Leibler [15]. [16], [17], en imágenes multitemporales SPOT pancromáticas de alta resolución espacial. Una vez obtenidas las imágenes, resultado de la aplicación de estos Indices de Cambios, se unen para generar una imagen multibanda, a la que posteriormente se aplica un clasificador supervisado basado en Máquinas de Soporte 
Vectorial (SVM). para obtener el mapa de cambios o imagen binaria final. Esta clasificación basada en SVM, sustituye el largo proceso de prueba y error que supondría elegir el método más adecuado de umbralización, típico en métodos no supervisados.

La organización del trabajo es la siguiente: en la Sección II se aborda el problema de obtención del mapa de cambios, de forma teórica, especificando las características de los Indices de Cambios propuestos; en la sección III se introducen las características del clasificador binario basado en SVM; en la sección IV se explica la metodología de Detección de Cambios establecida; en la Sección V se muestra el caso de estudio y un análisis de los resultados obtenidos: y finalmente. se pueden encontrar las conclusiones en la Sección VI.

\section{Mapa de Cambios}

Para abordar el problema de Detección de Cambios de una manera precisa, se expresará la secuencia de imágenes multitemporales como $\left\{I_{*}, I_{2}, \ldots I_{M}\right\}$ (típicamente $M=2$ ). en las que cada pixel del mapa de imagen, localizado por sus coordenadas $x \in R^{l}(l=2$, en imágenes registradas por sensores de satélite), es representado por una intensidad $I(x) \in R^{k}$, donde $k=1$ en imágenes codificadas en niveles de gris (o niveles digitales).

Un algoritmo básico para detectar cambios. toma la secuencia de imágenes como entrada y genera una imagen binaria $B: R^{I} \rightarrow[0,1]$ denominada mapa de cambios que identifica las regiones cambiantes de acuerdo con la siguiente regla:

$B(x)= \begin{cases}1, & \text { si hav un cambio significativo en } x \text { de } I_{M} \\ 0, & \text { en los demas casos. }\end{cases}$

Tradicionalmente, en una primera etapa se genera la imagen Indice de Cambios, $I D(x)$, y mediante un proceso de umbralización, en una segunda etapa, se obtiene el mapa de cambios (o imagen binaria), $B(x)$, según:

$B(x)= \begin{cases}1, & \text { si }|I D(x)| \geq \tau \\ 0, & \text { en los demas casos. }\end{cases}$

El valor del umbral es seleccionado, en la mayoria de los casos, de manera empírica [18]

\section{A. Indice de Cambios: Imagen Diferencia}

La Imagen Diferencia, $D(x)$, se obtiene por sustracción píxel a pixel de los niveles digitales de las imágenes multitemporales. La ecuación 3 muestra la expresión matemática que se utiliza para el cálculo del valor de la diferencia:

$$
D(x)=I_{2}(x)-I_{1}(x)
$$

\section{B. Indice de Cambios: Imagen Cociente}

La Imagen Cociente, $R(x)$, se obtiene por división píxel a píxel de los niveles digitales de las imágenes multitemporales. La ecuación 4 muestra la expresión matemática que se utiliza para el cálculo del valor del cociente:

$$
R(x)=I_{2}(x) / I_{1}(x)
$$

\section{Indice de Cambios: Imagen Distancia de Kullback-Leibler}

En este caso se debe asumir como premisa inicial que se considera que un pixel ha cambiado, si su distribución estadística cambia de una imagen a la otra $\left(I_{1}, I_{2}\right)$. Para cuantificar este cambio, se utiliza como medida un indice escalar, la Divergencia de Kullback-Leibler, que mapea las dos distribuciones estadísticas estimadas para cada imagen (multitemporal) y en su versión simétrica puede ser denominada como Distancia de Kullback-Leibler [15] según se obtiene mediante la siguiente expresión (5):

$D\left(I_{1}, I_{2}\right)=D\left(I_{2}, I_{1}\right)=K\left(I_{2} / I_{1}\right)+K\left(I_{1} / I_{2}\right)$

Este índice se puede calcular como la entropia entre las dos funciones de densidad de probabilidad de las dos imágenes. Para poder comparar dichas funciones deben ser conocidas y, para ello, se debe realizar una exploración de la imagen, mediante ventanas de pequeñas dimensiones, usando modelos de estadísticos locales. Para una función de densidad de probabilidad gaussiana con parámetros locales de media $\mu$ y varianza $\sigma^{2}$, el detector aplicado en cada ventana de dimensión seleccionada, es calculado recorriendo toda la imagen, utilizando la siguiente operación focal (6):

detector $=\frac{\sigma_{1}^{4}+\sigma_{2}^{4}+\left(\mu_{1}-\mu_{2}\right)^{2}\left(\sigma_{1}^{2}+\sigma_{2}^{2}\right)^{2}}{2 \sigma_{1}^{2} \sigma_{2}^{2}}-1$

\section{CLASIFICADOR BINARIO BASADO EN SVM}

Las Máquinas de Soporte Vectorial (SVM) son algoritmos de aprendizaje supervisado [19], basados en la teoría del aprendizaje estadístico, considerados como algoritmos heurísticos [20]. Conceptualmente, la Máquina implementa la siguiente idea: un conjunto de vectores no lineales mapea un espacio de características de elevadas dimensiones, para construir una superficie de decisión lineal. Las caracteristicas especiales de esta superficie de decisión aseguran un óptimo aprendizaje de la Máquina. Básicamente y particularizando en clasificación de imágenes. el objetivo de las SVM es determinar un hiperplano óptimo que separa dos clases (clasificador binario) utilizando datos de entrenamiento.

Así, sea un conjunto de patrones de entrenamiento etiquetados de acuerdo con la expresión (7):

$\left(y_{1}, x_{1}\right) \ldots \ldots,\left(y_{i}, x_{i}\right) \quad y_{i} \in\{-1,1\}$

Para ser linealmente separables deben existir un vector $w$ y un escalar $b$ tal que se cumplan las siguientes inecuaciones (8).

$w \cdot x_{i}+b \geq-1$ if $y_{i}=1$ )

$w \cdot x_{i}+b \leq-1$ if $\left.y_{i}=-1\right\}$

para todo el conjunto de entrenamiento. Entonces se pueden expresar las inecuaciones según (9):

$y_{1}\left(w \cdot x_{i}+b\right) \geq 1, \quad i=1, \ldots \ldots, l$

Como pueden existir muchos hiperplanos que proporcionen una separación efectiva se selecciona el que provee la separación óptima mediante la maximización de la distancia entre sí mismo y los planos que representan las dos clases. El hiperplano óptimo se representa por la expresión (10):

$w_{0} \cdot x_{i}+b_{0}=0$

siendo el único que separa los datos de entrenamiento con un margen máximo (Fig. 1), determinando la dirección $w / w \mid$ en la 
que la distancia entre la proyección de los vectores de entrenamiento de las dos clases es máxima.

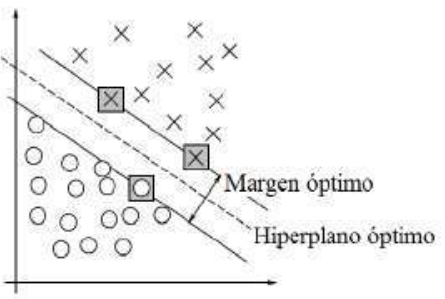

Figura 1. Ejemplo de separación en dos clases en un espacio de características bidimensional. Los vectores soporte están sombreados en gris. Las clases son reconocidas mediante aspas y círculos. Imagen basada en [19]

Esta distancia $\rho(w, b)$ viene dada por la expresión (11):

$\rho(w, b)=\min _{\{x: y=1\}} \frac{x \cdot w}{|w|}-\max _{\{x: y=-1\}} \frac{x \cdot w}{|w|}$

E1 hiperplano óptimo $\left(w_{0}, b_{0}\right)$ es el argumento que maximiza la distancia según (12):

$\rho\left(w_{0}, b_{0}\right)=\frac{2}{\left|w_{0}\right|}=\frac{2}{\sqrt{w_{0} \cdot w_{0}}}$

lo que significa que es el único que minimiza $w \cdot w$ bajo la condición impuesta en la ecuación (8). Los vectores $x_{i}$ para los que se cumple $y_{i}\left(w \cdot x_{i}+b\right)=1$ se denominan vectores soporte.

Las SVM utilizan las funciones Kernel definidas previamente, para trasformar las fronteras (entre las clases) de decisión no lineales (clases no separables), en lineales (clases separables) [21].

En el caso de este estudio, los kernel [21] que se utilizan son los siguientes (13)-(16):

Lineal: $\quad K\left(x_{i}, x_{j}\right)=x_{i}^{T} x_{j}$

Polinomial: $K\left(x_{i}, x_{j}\right)=\left(\gamma x_{i}^{T} x_{j}+r\right)^{d}, \gamma>0$

Función base radial: $K\left(x_{i}, x_{j}\right)=$

$$
=\exp \left(-\gamma\left\|\left(x_{i} x_{j}\right)\right\|^{2}, \gamma>0\right.
$$

Sigmoide: $\quad K\left(x_{i}, x_{j}\right)=\tan H\left(\gamma x_{i}^{T} x_{j}+r\right)$

donde $\gamma$ es la anchura de la función kernel, $d$ es el grado del polinomio y $r$ es el término bias de la función kernel.

Las SVM se pueden aplicar a la DC cuando el cambio y no_cambio se consideran como un problema de clasificación binaria. Se trata de un clasificador que se ajusta a la exigencia del problema de detección de cambios perfectamente, sobre todo, cuando el número de píxeles de las categorías cambio/ no_cambio a detectar, es pequeño en comparación con las dimensiones de imagen completa [21].

Estos clasificadores, comparados con otros, son muy eficientes desde diferentes perspectivas: utilizando conjuntos de entrenamiento pequeños, se obtienen muy buenos resultados; el proceso de aprendizaje es un proceso matemático que permite obtener el mejor clasificador; el tiempo de entrenamiento es predecible y relativamente corto; $\mathrm{y}$, por último, posee una alta velocidad de ejecución en la clasificación de grandes conjuntos de datos [20].

\section{METODOLOGIA}

\section{A. Caracteristicas de la imagen}

Se trata de dos escenas multitemporales registradas por el satélite SPOT5 el 24 Julio de 2005 y el 19 Julio de 2008 de dimensiones de $5846 \times 5760$ pixeles, situadas en la Comunidad de Madrid (España) con coordenadas geográficas UTM, 445584.730E y 4474899.400N (zona 30) (Fig. 2). El satélite SPOT proporciona imágenes con una banda pancromática, PAN, de resolución espacial de 2.5 (o 5 ) metros; tres bandas, $\mathrm{B} 1, \mathrm{~B} 2$ y B3, con resolución espacial de 10 metros y una banda situada en el Infrarrojo Medio, IRM, con una resolución espacial de 20 metros. Las características espectrales [22] de dichas bandas se representan en la Fig. 3.
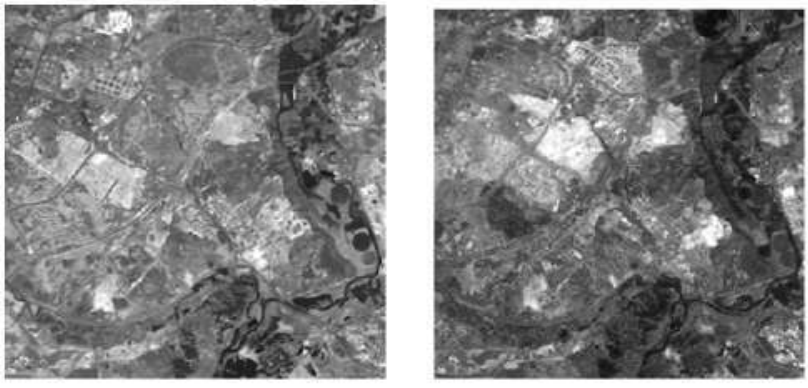

Figura 2. Bandas PAN-SPOT5 correspondientes al área de estudio.

Para este estudio, se selecciona la banda PAN en ambas fechas (Fig. 3), porque es la que presenta la mayor resolución espacial ( 2.5 metros). La resolución radiométrica es de 8 bits, en consecuencia la respuesta espectral de los diferentes píxeles está codificada entre 0 y 255 niveles de gris (niveles digitales).

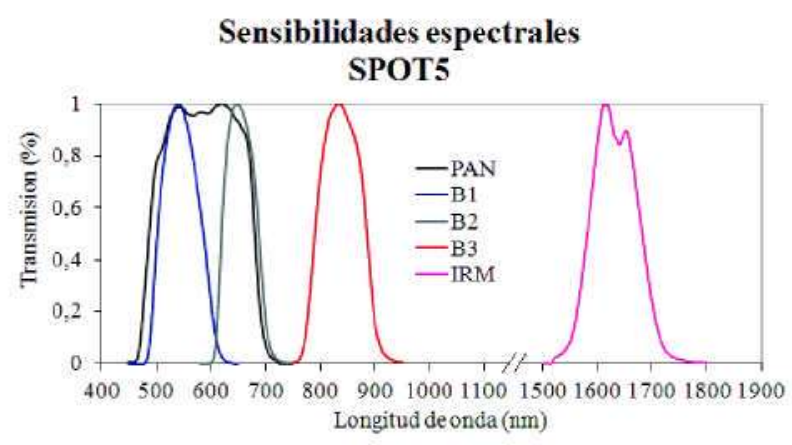

Figura 3. Sensibilidades espectrales en SPOT5

\section{B. Propuesta metodológica de Detección de Cambios}

En esta sección se propone un nuevo método (Fig. 4) de obtención de mapas de cambios mediante procedimientos no supervisados de determinación de imágenes Indice de Cambios. A continuación se realiza un análisis conjunto posterior, uniendo estas bandas en una única imagen multibanda, que será introducida en un proceso de clasificación binaria supervisada, basada en SVM para obtener el mapa de cambios. Esta propuesta evita el proceso de umbralización, que presenta un elevado coste computacional adicional. 


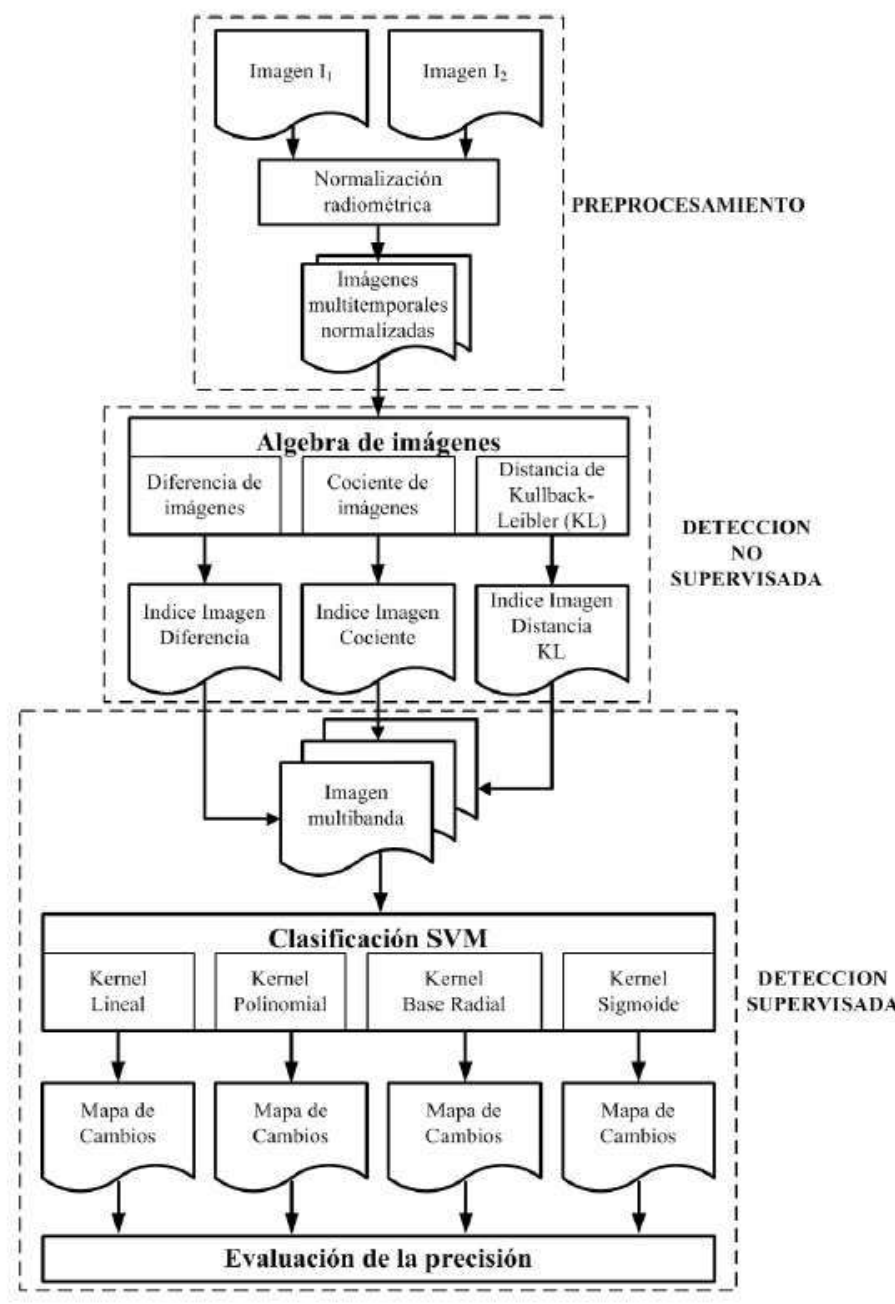

Figura 4. Diagrama metodológico del proceso de Detección de Cambios.

\section{1) Preprocesamiento de las imágenes.}

Debido a las variaciones en las condiciones atmosféricas y parámetros del sensor del satélite, durante el proceso de adquisición de los datos, las imágenes de la misma zona geográfica, que han sido adquiridas en diferentes fechas, deben ser normalizadas para poder compararlas. Para ello se han desarrollado una gran variedad de técnicas de normalización radiométrica relativa [23]. Aunque los datos SPOT se suministran con corrección radiométrica, se requiere normalizar las imágenes para trabajar con valores de radiancia o reflectancia [24].

\section{2) Detección no supervisada: Cálculo de las imágenes Indices de Cambios.}

Para la obtención de estas imágenes, se programan y aplican sobre las bandas PAN de las dos fechas los procedimientos algebraicos basados en el cálculo de la Imagen Diferencia, Imagen Cociente y la Imagen Distancia de Kullback-Leibler, para las diferentes dimensiones de ventana ( $3 \times 3,7 \times 7,9 \times 9$ y $11 \times 11$ píxeles), según se ha comentado en la sección $\Pi$.

\section{3) Detección supervisada: Fusión de información} multifuente. Clasificación supervisada.

Los mapas binarios obtenidos a partir de las tres fuentes de Indices de Cambios, en los pasos anteriores, son unidos para formar una imagen multibanda, a la que se somete a un proceso de clasificación supervisada mediante SVM con los cuatro kernel (13) (14) (15) (16).

Los parámetros comunes [22] que se establecen para todos los kernel seleccionados son: el parámetro de penalización, el nivel piramidal y el valor umbral de probabilidad de clasificación. El parámetro de penalización se establece en todos los casos a su valor máximo, 1000 , lo que obliga a todos los píxeles de entrenamiento a converger a una clase. El nivel piramidal se ajusta a un valor de cero para todos los kernel, por lo que la imagen se procesa con su resolución espacial, lo que puede provocar una disminución de la velocidad de proceso. Si se procesara con una resolución menor, mejoraría la velocidad de proceso. A la probabilidad de clasificación se aplica un umbral de cero, restricción obligada para no obtener pixeles sin clasificar.

\section{4) Determinación de la precisión}

Para evaluar los resultados de clasificación y, por lo tanto, la bondad del mapa de clasificación cambio/no_cambio, se seleccionan áreas de test, cuyos píxeles contienen la información denominada verdad-terreno y que se supervisan visualmente sobre las imágenes de las dos fechas.

Una vez que la imagen binaria procedente de la clasificación se ha obtenido, se comparan sus resultados con los procedentes de los píxeles test.

Para realizar un análisis preciso se construyen las matrices de error con dimensiones de $2 \times 2$, cambio/no_cambio, y se calcula la precisión global, las de usuario y productor (relacionadas con el error por comisión y por emisión), y el coeficiente Kappa [25].

El error por omisión aparece cuando un píxel que pertenece a determinada categoría o clase, no resulta clasificado en dicha clase (falso negativo). Se plantea como riesgo del productor. Sin embargo, el error por comisión ocurre cuando un píxel resulta clasificado en una categoria y en realidad pertenece a otra (falso positivo). En este caso se plantea como riesgo del usuario.

La precisión global se calcula como el número total de pixeles de test correctamente clasificados, que coinciden con la diagonal principal en una matriz de error, dividido por el número total de píxeles test. Y el coefíciente Kappa se obtiene mediante la siguiente expresión (17):

$$
K=\frac{N \sum_{i=1}^{r} x_{i i}-\sum_{i=1}^{r} x_{i+} x_{+i}}{N^{2}-\sum_{i=1}^{r} x_{i+} x_{+i}}
$$

donde $r$ es el número de filas de la matriz de error, $x_{i i}$ es el número de observaciones o píxeles test en la fila $i$ y en la columna $i ; x_{i+}$ y $x_{+i}$ son los totales marginales en la fila $i$ y en la columna $i$, respectivamente, y $N$ es el número total de observaciones.

Desde el punto de vista de la metodología de Detección de Cambios se hace necesario evaluar si la detección ha sido o no correcta. Para ello se calculan los siguientes valores con respecto al total de los píxeles test, $N$ :

- Verdaderos positivos, VP: es el número de píxeles cambiados que se detectan correctamente y su proporción será $P_{V P}=V P / N$. 
- Falsos positivos, FP o falsas alarmas: es el número de píxeles sin cambio que se detectan incorrectamente, como cambiados, y su proporción será $P_{F P}=F P / N$.

- Verdaderos negativos, $V N$ : es el número de píxeles sin cambio que se detectan correctamente y su proporción será $P_{V N}=V N / N$.

- Falsos negativos, $F N$ : es el número de píxeles cambiados que se detectan incorrectamente como no_cambio y su proporción será $P_{F N}=F N / N$.

Por tanto, el rendimiento de la metodología propuesta puede ser medida en términos de la detección correcta, $P_{D C}, \mathrm{y}$ de detección falsa, $P_{D F}$, que son, respectivamente, definidas como (18) (19):

$P_{D C}=P_{V P}+P_{V N}$

$P_{F D}=P_{F P}+P_{P N}$

\section{RESULTADOS}

Las imágenes Indices de Cambios resultantes se muestran en la Fig. 5, siendo una ventana de $11 \times 11$ píxeles la seleccionada como óptima en el caso del detector para la Distancia de Kullback-Leibler.

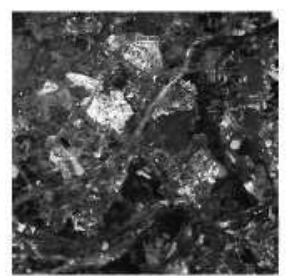

Imagen Diferencia

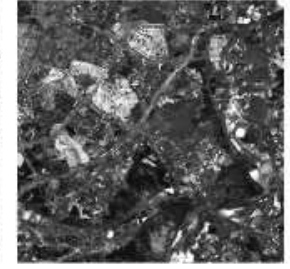

Imagen Cociente

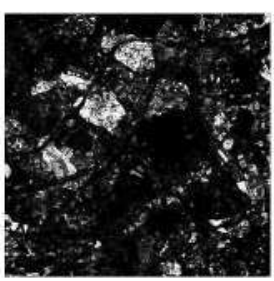

Imagen Distancia de KL
Figura 5. Imágenes Indices de Cambios de la Imagen Diferencia, la Imagen Cociente y la Imagen de Kullback-Leibler.

El conjunto de datos de estas tres imágenes es supervisado para seleccionar las áreas de la fase de entrenamiento del clasificador. Para el entrenamiento, un experto realiza una selección de 103178 píxeles $(3 \%$ del total) de cambio/no_cambio, recorriendo toda la imagen. El proceso de clasificación se lleva a cabo mediante el software ENVI 4.5 (Environment for Visualizing Images) [26], aplicando SVM con los cuatro kernel comentados en el apartado III.

Con objeto de construir las matrices de error del proceso de obtención de los mapas de cambios mediante clasificación, se seleccionan 11084 píxeles de cambio/no_cambio como píxeles de test. Las matrices se muestran en la Tabla I.

Derivados de los resultados obtenidos y reflejados en la Tabla I, se obtienen los valores de las precisiones y coeficiente Kappa de las clasificaciones realizadas mediante SVM, representados en la Tabla II.

De los resultados reflejados en las Tablas anteriores, el clasificador SVB base radial es el que presenta los mejores resultados respecto a la evaluación de errores de clasificación. En los cuatros casos se obtienen valores superiores al $98 \%$ en la precisión global.

La imagen binaria correspondiente al mapa de cambios obtenido en este caso se muestra en la Fig. 6.

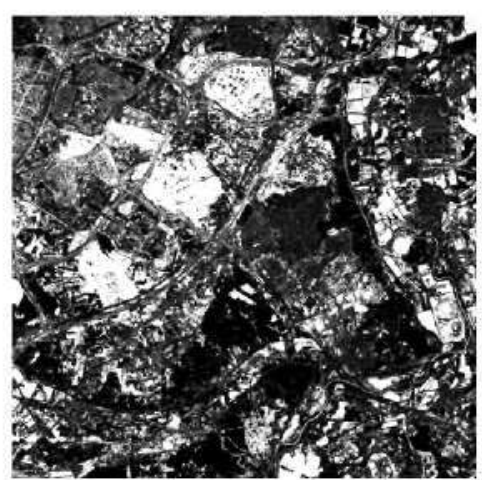

Figura 6. Imagen binaria correspondiente al mapa de cambios con zonas de cambio (de color blanco) y zonas de no cambio (de color negro), obtenido mediante el clasificador SVM base radial.

TABLA I. MATRICES DE ERROR PARA LAS CLASES CAMBIO (C) Y NO_CAMBIO (NC) EN LAS CLASIFICACIONES CON SVM. C TEST Y NC TEST SON LOS PIXXELES DE LAS AREAS SUPERVISADAS (VERDAD-TERRENO). $\mathrm{C}_{\mathrm{CL}}$ Y NC $\mathrm{NC}_{\mathrm{CL}}$ SON LOS PÍELES RESULTANTES EN LA CLASIFICACIÓN, CORRESPONDIENTES A LASAREAS TEST.

\begin{tabular}{|c|c|c|c|c|c|}
\hline \multirow{2}{*}{ Tipo SVM } & \multicolumn{3}{|c|}{ MATRICES } & \multicolumn{2}{|c|}{$\begin{array}{c}\text { ERRORES } \\
\text { EN CLASIFICACION }\end{array}$} \\
\hline & & $\mathrm{C}_{\text {TEST }}$ & $\mathrm{NC}_{\text {TEST }}$ & $\begin{array}{c}\text { Error por } \\
\text { Comisión }(\%)\end{array}$ & $\begin{array}{c}\text { Error por } \\
\text { Omisión }(\%)\end{array}$ \\
\hline \multirow{2}{*}{ SVM lineal } & \multirow{2}{*}{$\begin{array}{l}\mathrm{C}_{\mathrm{CL}} \\
\mathrm{NC}_{\mathrm{CL}}\end{array}$} & \multirow{2}{*}{$\begin{array}{c}7013 \\
23\end{array}$} & \multirow{2}{*}{$\begin{array}{c}169 \\
3879 \\
\end{array}$} & 2.35 & 0.33 \\
\hline & & & & 0.59 & 4.17 \\
\hline \multirow{2}{*}{$\begin{array}{c}\text { SVM } \\
\text { polinomial }\end{array}$} & \multirow{2}{*}{$\begin{array}{l}\mathrm{C}_{\mathrm{CL}} \\
\mathrm{NC}_{\mathrm{CL}}\end{array}$} & \multirow{2}{*}{$\begin{array}{c}7013 \\
23\end{array}$} & \multirow{2}{*}{$\begin{array}{c}141 \\
3907\end{array}$} & 1.97 & 0.33 \\
\hline & & & & 0.59 & 3.48 \\
\hline \multirow{2}{*}{$\begin{array}{l}\text { SVM base } \\
\text { radial }\end{array}$} & \multirow{2}{*}{$\begin{array}{l}\mathrm{C}_{\mathrm{CL}} \\
\mathrm{NC}_{\mathrm{CL}}\end{array}$} & \multirow{2}{*}{$\begin{array}{c}7013 \\
23\end{array}$} & \multirow{2}{*}{$\begin{array}{c}132 \\
3916\end{array}$} & 1.85 & 0.33 \\
\hline & & & & 0.58 & 3.26 \\
\hline \multirow{2}{*}{$\begin{array}{c}\text { SVM } \\
\text { sigmoide }\end{array}$} & \multirow{2}{*}{$\begin{array}{l}\mathrm{C}_{\mathrm{CL}} \\
\mathrm{NC}_{\mathrm{CL}}\end{array}$} & 7013 & \multirow{2}{*}{$\begin{array}{c}192 \\
3856 \\
\end{array}$} & 2.66 & 0.33 \\
\hline & & 23 & & 0.59 & 4.74 \\
\hline
\end{tabular}

TABLA II. PRECISIONES Y COEFICIENTE KAPPA PARA LAS CLASES CAMBIO (C) Y NO CAMBIO (NC) EN LAS CLASIFICACIONES CON SVM.

\begin{tabular}{c|cc|cc|c}
\hline \multirow{2}{*}{ Tipo SVM } & \multicolumn{2}{|c|}{$\begin{array}{c}\text { Precisión del } \\
\text { Productor (\%) }\end{array}$} & \multicolumn{2}{|c|}{$\begin{array}{c}\text { Precisión del } \\
\text { Usuario (\%) }\end{array}$} & \multirow{2}{*}{$\begin{array}{c}\text { Precisión } \\
\text { global (\%) } \\
\text { Coeficiente } \\
\text { Kappa }\end{array}$} \\
\cline { 2 - 5 } & $\mathrm{C}$ & $\mathrm{NC}$ & $\mathrm{C}$ & $\mathrm{NC}$ & \multicolumn{2}{|c|}{$\begin{array}{c}\text { Non } \\
\text { SVM lineal }\end{array}$} & 99.67 & 95.83 & 97.65 & 99.41 & $98.27 / 0.96$ \\
\hline $\begin{array}{c}\text { SVM } \\
\text { polinomial }\end{array}$ & 99.67 & 96.52 & 98.03 & 99.41 & $98.52 / 0.97$ \\
\hline $\begin{array}{c}\text { SVM base } \\
\text { radial }\end{array}$ & 99.67 & 96.74 & 98.15 & 99.42 & $98.60 / 0.97$ \\
\hline $\begin{array}{c}\text { SVM } \\
\text { sigmoide }\end{array}$ & 99.67 & 95.26 & 97.34 & 99.41 & $98.06 / 0.96$ \\
\hline
\end{tabular}

TABLA III. VALORES DE RENDIMIENTO DE LA METODOLOGIA EN TÉRMINOS DETECCIÓN CORRECTA $\left(\mathrm{P}_{\mathrm{DC}}\right)$ Y DETECCIÓN FALSA $\left(\mathrm{P}_{\mathrm{FD}}\right)$.

\begin{tabular}{c|cc|c|cc|c}
\hline \hline Tipo SVM & $\mathrm{P}_{\mathrm{VP}}$ & $\mathrm{P}_{\mathrm{VN}}$ & $\mathrm{P}_{\mathrm{DC}}$ & $\mathrm{P}_{\mathrm{FP}}$ & $\mathrm{P}_{\mathrm{FN}}$ & $\mathrm{P}_{\mathrm{FD}}$ \\
\hline SVM lineal & 0.63 & 0.35 & 0.98 & 0.015 & 0.021 & 0.036 \\
\hline $\begin{array}{c}\text { SVM } \\
\text { polinomial }\end{array}$ & 0.63 & 0.35 & 0.98 & 0.013 & 0.021 & 0.034 \\
\hline $\begin{array}{c}\text { SVM base } \\
\text { radial }\end{array}$ & 0.63 & 0.35 & 0.98 & 0.012 & 0.021 & 0.033 \\
\hline $\begin{array}{c}\text { SVM } \\
\text { sigmoide }\end{array}$ & 0.63 & 0.34 & 0.97 & 0.017 & 0.021 & 0.038 \\
\hline
\end{tabular}

En cuanto al rendimiento de la metodología de DC propuesta se puede indicar, según muestra la Tabla III, que en todos los casos los valores de $\mathrm{P}_{\mathrm{DC}}$ están muy próximos a 1 y los de $\mathrm{P}_{\mathrm{FD}}$ se encuentran muy próximos a 0 , lo que indica un alto rendimiento con elevada corrección de la DC en todos los casos. 


\section{CONCLUSIONES}

En este trabajo se ha presentado una metodologia de Detección de Cambios semi-supervisada que presenta un alto rendimiento en términos de detección correcta. La propuesta de utilizar clasificadores basados en Máquinas de Soporte Vectorial para obtener las imágenes binarias correspondientes a los mapas de cambios. produce resultados muy satisfactorios debido a la precisión global del $98 \%$ alcanzada en la determinación de las clases cambio/no_cambio. Como era de esperar, se ha necesitado un conjunto pequeño de patrones para el entrenamiento ( $3 \%$ del total de pixeles) de los clasificadores basados en los cuatro diferentes kernel.

Así mismo, se puede concluir que la programación y utilización del índice basado en la Distancia de KullbackLeibler, no muy frecuente entre métodos clásicos [4], [5], en este ámbito de investigación, ha contribuido en gran medida debido a su robustez, junto con los dos Indices de Cambios estándar, diferencia y cociente, a los buenos resultados obtenidos.

\section{REFERENCIAS}

[1] A. Singh, "Digital clange detection tecluniques using remotely-sensed data". Int. J. Remote Sensing, vol. 10, 100. 6, pp. 989-1003, 1989.

[2] R. Radke, S. Andra, O. Al-Kofahi, and B. Roysam. "Image change detection algorithms: A systematic survey", IEEE Trans. Inage Process., vol. 14, no. 3, pp. 294-307. 2005.

[3] J. L. A. Samatelo and E. O. T. Salles, "A New Change Detection Algorithm for Visual Surveillance System". IEEE Latin America Transactions, vol. 10, no. 1. 2012.

[4] P. R. Coppin, I. Jonckheere, and K. Nachaerts, "Digital change detection in ecosystem monitoring: A review", Int. J. Remote Sens., vol. 25, no. 9, pp. 1565-1596, 2004.

[5] D. Lu, P. Mausel, E. Brondizio, and E. Moran, "Change detection techniques", Int. J. Remote Sens., vol. 25, no. 12, pp. 2365-2407. 2004.

[6] D. Fernández-Prieto and M. Marconcini. "A Novel Partially Supervised Approach to Targeted Change Detection". IEEE Trans. on Geoscience and Remote Sensing, vol. 49, no. 12, 2011.

[7] M. Volpi, D. Tuia, F. Bovolo, M. Kanevski and L. Bruzzone, "Supervised change detection in VHR images using contextual information and support vector machines". Int. J. of Applied Earth Observation and Geoinformation, vol. 20. pp. 77-85, 2013.

[8] X. Yang and C. P. Lo, "Relative radionetric normalization performance for change detection from multi-date satellite images". Photogramm. Eng. Remote Sens., vol. 66, pp. 967-980, 2000.

[9] M. L. F. Velloso. F. J. de Souza and M. Simoes. "Improved radiometric normalization for land cover change detection: An automated relative correction with artificial neural network", in Proc. IEEE Int. Geosci. Remote Sens. Symp., pp. 3435-3437, 2002.

[10] S. Marchesi. F. Bovolo, and L. Bruzzone. "A context-sensitive technique robust to registration noise for change detection in VHR multispectral images". IEEE Trans. Image Process., vol. 19, no. 7, pp. 1877-1889, 2010.

[11] M. Ding. Z. Tian, Z. Jin, M. Xu, and C. Cao, "Registration using robust kernel principal component for object-based change detection",IEEE Geosci. Remote Sens. Lett., vol. 7, no. 4, pp. 761-765, 2010.

[12] F. Bovolo, L. Bruzzone, and S. Marchesi, "Analysis and adaptive estimation of the registration noise distribution in nultitemporal VHR images", IEEE Trans. Geosci. Renote Sens., vol. 47, no. 8. pp. 2658$2671,2009$.

[13] S. Le Hegarat-Mascle. R. Seltz L. Hubert-Moy, S. Corgne, N. Stach, "Performance of change detection using remotely sensed data and evidential fusion: Conparison of three cases of application". Int. J. Remote Sens. Vol. 27, no. 16, pp. 3515-3532, 2006.

[14] I. Molina, E. Martinez, A. Arquero, G. Pajares and J. Sanchez, "Evaluation of a Change Detection Methodology by Means of Binary Thresholding Algorithms and Informational Fusion Processes", Sensors vol. 12, pp. 3528-3561, 2012.

[15] J. Inglada and G. Mercier, "A New Statistical Similarity Measure for Change Detection in Multitemporal SAR images and Its Extension to
Multiscale Change Analysis", IEEE Trans. on Geosci. Remote Sens., vol. 45, no. $5,2007$.

[16] Z. Chen "Probabilistic Representation of Structural Integrity of Urban Buildings in Remotely Sensed Images", in Proc. IEEE International Symposium of Geoscience and Remote Sensing. IGARSS 2008, vol.4, pp. 315-318, 2008.

[17] X. Zeng and T. S. Durrani, "Image Change Detection using Copulas", In Proc. International Conference on Signal Processing. ICSP 2008, pp. 909-913, 2008.

[18] P. Rosin and E. Ioannidis, "Evaluation of global inage thresholding for change detection", Pattern Recognit. Lett., vol. 24, no. 14, pp. 23452356,2003

[19] C. Cortes and V. Vapnik "Support vector networks", Machine Learning. vol. 20. pp. 273-297, 1995.

[20] V.N. Vapuik, The Noture of Statistical Learning Theory: Springer Verlag, 2000.

[21] P. K. Srivastava, D. Han, M. A. Rico-Ramirez, M. Bray, T. Islam, "Selection of classification techniques for land use/land cover change investigation". Advances in Space Research, vol. 50. pp. 1250-1265, 2012.

[22] http:/wwwastrium-geo.com/es/441-imagenes-por-satelite-spot (acceso Febrero 2014)

[23] S. Marchesi, F. Bovolo, and L. Bruzzone, "A context-sensitive teclunique robust to registration noise for change detection in VHR multispectral images". IEEE Trans. Image Process, vol. 19, no. 7. pp. 1877-1889. 2010.

[24] M. El Hajj, A. Bégué, B. Laftance, O. Hagolle, G. Dedieu, and M. Rumeau, "Relative radiometric normalization and atmospheric correction of a SPOT 5 time series", Sensors, vol. 8, no. 4, pp. 2774 2791,2008

[25] R. G. Congalton and K. Green, Assessing the Accuracy of Remotely Sensed Data - Principles and Practices. $2^{\text {n }}$ ed. CRC Press. Taylor \& Francis Group. 2009.

[26] http:/www.exelisvis.com/ProductsServices/ENVI/ENVI.aspx (acceso Febrero 2014) 